\title{
Expression of the Adhesion Molecule Spermatogenic Immunoglobulin Superfamily (SgIGSF) in Mouse Tissues
}

\author{
Tomohiko Wakayama $^{1}$, Hiroyuki Koami ${ }^{1}$, Miyuki Yamamoto ${ }^{1}$ and Shoichi Iseki ${ }^{1}$ \\ ${ }^{1}$ Department of Histology and Embryology, Graduate School of Medical Science, Kanazawa University, 13-1 Takara-machi, \\ Kanazawa 920-8640, Japan
}

Received November 15, 2004; accepted December 14, 2004

\begin{abstract}
Spermatogenic immunoglobulin superfamily (SgIGSF) is an adhesion molecule originally isolated from adult mouse testis. In the testis, SgIGSF is expressed specifically in spermatogenic cells and may be involved in spermatogenesis. SgIGSF may also be involved in synapse formation and tumor suppression. In the present study, we examined the expression and cellular localization of SgIGSF in the entire adult mouse organs and tissues using Western blot analysis and immunohistochemistry at light and electron microscopic levels. Western blot analysis revealed that SgIGSF is expressed not only in the testis but also in the liver, lung, and nervous system

including the cerebrum, cerebellum and sciatic nerve. The nervous system as well as testis showed multiple immunoreactive bands ranging from 45 to 100 $\mathrm{kDa}$, whereas the liver and lung showed a single $100 \mathrm{kDa}$ band. Immunohistochemistry demonstrated that in the nervous system, SgIGSF is localized to the membranes of synapses, axons and Schwann cells. In contrast, in the lung and liver SgIGSF was localized to the membranes of apposing respiratory epithelial cells, hepatocytes and biliary epithelial cells. These results suggested that SgIGSF plays multiple physiological roles in the adult mouse as an adhesion molecule.
\end{abstract}

Key words: SgIGSF, immunoglobulin superfamily, adhesion molecule, western blot, immunohistochemistry

\section{Introduction}

Cell-to-cell or cell-to-extracellular matrix adhesion is crucial for initiation and maintenance of cell contacts that are essential for specialized tissue structure and function in multicellular organisms $[6,8]$. Cell recognition and adhesion are mediated by cell surface adhesion molecules that bind to proteins presented on the surface of neighboring cells or deposited in the extracellular matrix. In addition to the mechanical adhesive activity, there is evidence that these adhesion molecules can also modulate the intracellular signaling [1]. For example, $\beta$-catenin, which is a scaffolding protein associated with cadherin, plays a role as transcription factor in the nucleus [14]. Adhesion molecules have been classified into several groups on the basis of their primary structure, including the immunoglobulin super-

Correspondence to: Tomohiko Wakayama, M.D., Ph.D., Department of Histology and Embryology, Graduate School of Medical Science, Kanazawa University, 13-1 Takara-machi, Kanazawa 920-8640, Japan. E-mail: twaka@basic.m.kanazawa-u.ac.jp family (IGSF) [24], cadherin family [19, 25], integrin family [10], and selectin family [20]. These adhesion molecules may play pivotal roles in morphogenesis and organogenesis [6].

Molecules of the IGSF are characterized by the presence of $\mathrm{N}$-terminal immunoglobulin-like domains that are responsible for binding with each other or with other ligands [9]. The IGSF is consisted of 765 members in humans, 140 in flies, and 64 in worms according to the genome sequence research [5], and have essential roles in a wide variety of biological processes that involve cell-to-cell interactions [15]. IGSF often mediates intracellular signal transductions between extracellular ligands and second-messenger cascades [4, 16]. Many IGSF molecules have transmembrane domains and cytoplasmic domains, some of which have enzymatic characteristics. The IGSF molecules with cytoplasmic receptor tyrosine kinase or phosphatase domain exert their intracellular signaling influence directly through their enzymatic activity, while others do so by associating with and activating intracellular kinases. For example, activation of tyrosine kinases of the src family by Ig-ligand 
binding of neural cell adhesion molecules causes effects on the dynamics of cytoskeletons [4], providing an important link between cellular adhesion and cell-shape changes associated with the morphogenetic movements during embryonic development [17].

Spermatogenic immunoglobulin superfamily (SgIGSF) is an evolutionally conserved membrane glycoprotein belonging to the IGSF with putative 445 amino acid residues in the mouse [21]. SgIGSF was originally isolated from adult mouse testis and shown to be expressed in spermatogenic cells but not in Sertoli cells, suggesting that it plays roles in the cellular interactions during spermatogenesis. SgIGSF also shows different molecular weights due to N-glycosylation of the protein during postnatal development of the testis [23]. Recently, SgIGSF has also been reported by other names, such as synaptic cell adhesion molecule (SynCAM), which is expressed in the cultured neuron and responsible for synapse formation in cooperation with the glutamate receptor [2], or tumor suppressor gene lung cancer 1 (TSLC1), which causes suppression of malignancy in a human lung cancer cell line A549 [12].

To shed light on the biological significance of SgIGSF, it is essential to determine the systemic expression and distribution of SgIGSF in physiological conditions. By use of Northern hybridization analysis, we demonstrated earlier that SgIGSF mRNA is expressed abundantly in the brain, testis and epididymis and weakly in the liver and kidney of mice [21]. Also, by use of a specific anti-SgIGSF antibody, we demonstrated that SgIGSF protein is localized to the plasma membrane of spermatogenic cells in the mouse testis [23]. In the present study, we explored the expression and cellular localization of SgIGSF in the entire organs and tissues of adult mice by use of Western blotting and immunohistochemistry with anti-SgIGSF antibody.

\section{Materials and Methods}

\section{Animals and tissue preparation}

Male and female Slc:ddY mice at the age of 10 weeks were purchased from Nippon SLC, Inc. (Hamamatsu, Japan) and grown under $12 \mathrm{hr}$ light:12 hr dark laboratory conditions. All procedures were performed according to the Guidelines for the Care and Use of Laboratory Animals at Kanazawa University. Mice were anesthetized with sodium pentobarbital by intraperitoneal injection, killed by bleeding from the right atrium, and perfused transcardially with cold physiological saline. For protein extraction, 19 organs and tissues (cerebrum, cerebellum, sciatic nerve, skeletal muscle, heart, thymus, spleen, lung, stomach, small intestine, liver, pancreas, kidney, adrenal gland, testis, ovary, epididymis, seminal vesicle, uterus) were dissected out from mice, frozen immediately in liquid nitrogen, and stored at $-80^{\circ} \mathrm{C}$ until use. To make tissue specimens for fluorescent immunohistochemistry, the animals were further perfused transcardially with $4 \%$ cold paraformaldehyde (Nacalai Tesque, Kyoto, Japan) in $0.1 \mathrm{M}$ phosphate-buffered saline (PBS) (pH 7.2). The tissues were dissected out, further im- mersed in the same fixative for $4 \mathrm{hr}$, dehydrated in a graded alcohol series for $24 \mathrm{hr}$, and embedded in paraffin. They were then cut into 4- $\mu$ m thick sections with a microtome (Leica Microsystems, Wetzlar, Germany) and mounted on glass slides. For electron-microscopic immunocytochemistry, the fixed tissues were immersed in PBS containing $30 \%$ sucrose overnight at $4{ }^{\circ} \mathrm{C}$ for cryoprotection. They were then frozen in PBS containing 30\% sucrose and 50\% OCT compound (Miles, Elkhart, IN, USA) and cut into $10-\mu \mathrm{m}$ sections using a cryostat (Leica Microsystems). The sections were mounted on poly-L-lysine (Sigma-Aldrich, St. Louis, MO, USA)-coated plastic slides.

\section{Western blot analysis}

Aliquots of cell lysates from mouse tissues containing $20 \mu \mathrm{g}$ proteins were subjected to SDS-PAGE and transferred to PVDF membranes (Bio-Rad Laboratories, Hercules, CA, USA). A polyclonal antibody against the carboxyl-terminal 15 peptides of mouse SgIGSF was raised in rabbits as reported previously [21]. The blots were incubated with antiSgIGSF antibody at $0.05 \mu \mathrm{g} / \mathrm{ml}$ for $1 \mathrm{hr}$ at room temperature (RT). After washing, the blots were further incubated with horseradish peroxidase-labeled anti-rabbit IgG antibody (DAKO, Glostrup, Denmark) at 1:5000 dilution for $1 \mathrm{hr}$ at RT. The immunoreaction was detected with X-ray film (Kodak, New Haven, CT, USA) after treatment of the blots with a chemiluminescent reagent ECL-plus (Amersham Pharmacia Biotech, Uppsala, Sweden). After stripping the immune products, the blots were re-probed with anti- $\alpha$ tubulin antibody (Sigma-Aldrich).

\section{Immunohistochemistry}

For fluorescent immunohistochemistry, paraffin sections were treated with $5 \%$ normal goat serum for $60 \mathrm{~min}$ to prevent non-specific antibody binding. Subsequently, the sections were incubated with anti-SgIGSF antibody at 1 $\mu \mathrm{g} / \mathrm{ml}$ overnight at $4^{\circ} \mathrm{C}$. After the sections were washed in PBS, the sites of immunoreaction were visualized by incubating the sections with Alxa 594-labelled anti-rabbit IgG (Molecular Probes, Eugene, OR, USA) for $1 \mathrm{hr}$ and observed under fluorescent microscopy using an Axiovert S100 microscope (Carl Zeiss, Oberkochen, Germany). To confirm the specificity of the immunoreaction, the antibody was preabsorbed with the peptide antigen used for the immunization ( $10 \mu \mathrm{g}$ of peptide for $1 \mu \mathrm{g}$ of $\operatorname{IgG})$ for $1 \mathrm{hr}$ at $4^{\circ} \mathrm{C}$ prior to use.

For electron-microscopic immunocytochemistry, the pre-embedding immunoreaction method was performed [22, 26]. Briefly, the cryostat sections on the plastic slides were incubated successively with anti-SgIGSF antibody overnight at $4{ }^{\circ} \mathrm{C}$, biotinylated anti-rabbit IgG antibody (Vector Laboratories, Burlingame, CA, USA) for $1 \mathrm{hr}$, horseradish peroxidase-conjugated streptavidin (DAKO) for $1 \mathrm{hr}$, and 3,3'-diaminobenzidine (Dojindo, Kumamoto, Japan) solution containing $\mathrm{H}_{2} \mathrm{O}_{2}$ for a few minutes. The immunostained sections were postfixed in $0.5 \% \mathrm{OsO}_{4}$ for $30 \mathrm{~min}$, stained with $1 \%$ uranyl acetate for $20 \mathrm{~min}$, dehydrated in graded eth- 
anol series and were embedded in Glycid ether 100 (Serva Feinbiochemica, Heidelberg, Germany). Ultrathin sections were cut with an ultramicrotome (LKB 4800A Ultrotome; LKB-Produkter, Stockholm, Sweden) and subjected to observation with a Hitachi H-700 electron microscope (Hitachi High-Technologies, Tokyo, Japan).

\section{Results}

\section{Western blot analysis}

Out of the 19 organs and tissues examined, the positive immunoreaction for SgIGSF was detected in the cerebrum, cerebellum, sciatic nerve, lung, liver and testis (Fig. 1). The testis showed the major immunopositive band of $120 \mathrm{kDa}$ and the minor bands of 90 and $100 \mathrm{kDa}$ representing the difference in the amount of glycosylation [21]. The cerebrum showed multiple immunopositive protein bands $45,60,65$, 70 and $100 \mathrm{kDa}$ and cerebellum 45, 60 and $65 \mathrm{kDa}$ in size, whereas the sciatic nerve showed a single $45 \mathrm{kDa}$ band. In contrast, both the lung and liver expressed a single $100-\mathrm{kDa}$ band.

\section{Immunohistochemistry}

Paraffin and frozen sections prepared from the organs and tissues in which SgIGSF was detected by Western blotting were subjected to immunohistochemistry. In the cerebrum and cerebellum, the fluorescence representing SgIGSF immunoreactivity of various intensity was distributed diffusely all over the cortex and medulla (Fig. 2A, C). When the antibody was absorbed with the specific blocking polypeptide, no immunostaining was observed in any cells or areas (Fig. 2B). Although the neuronal perikaryons such as located in the hippocampus and the dental gyrus of cerebrum and Purkinje layer of cerebellum were relatively low in the immunoreactivity, strong immunoreactivity was observed in many focal areas in the granular layer of cerebellum corresponding to the synaptic complexes (glomeruli). Electron microscopy demonstrated the immunoreactivity on the membranes of apposing neurons in the glomerules (Fig. 2D).

In the transverse section of sciatic nerve, SgIGSF immunoreactivity was localized to the boundary between the axon and myelin sheath and also to the outer surface of Schwann cells, whereas the inside of myelin sheath was immunonegative (Fig. 2E). In the immuno-electron microscopy, the immunoreactivity was localized to the axolemma and the membrane at the outer surface of Schwann cells, whereas it was difficult to determine if the membrane at the inner surface of myelin sheath was immunopositive for SgIGSF (Fig. 2F).

In the liver, SgIGSF immunoreactivity was localized to the boundaries between hepatocytes and between biliary epithelial cells (Fig. 2G). In the immuno-electron microscopy, the immunoreactivity was localized to the lateral

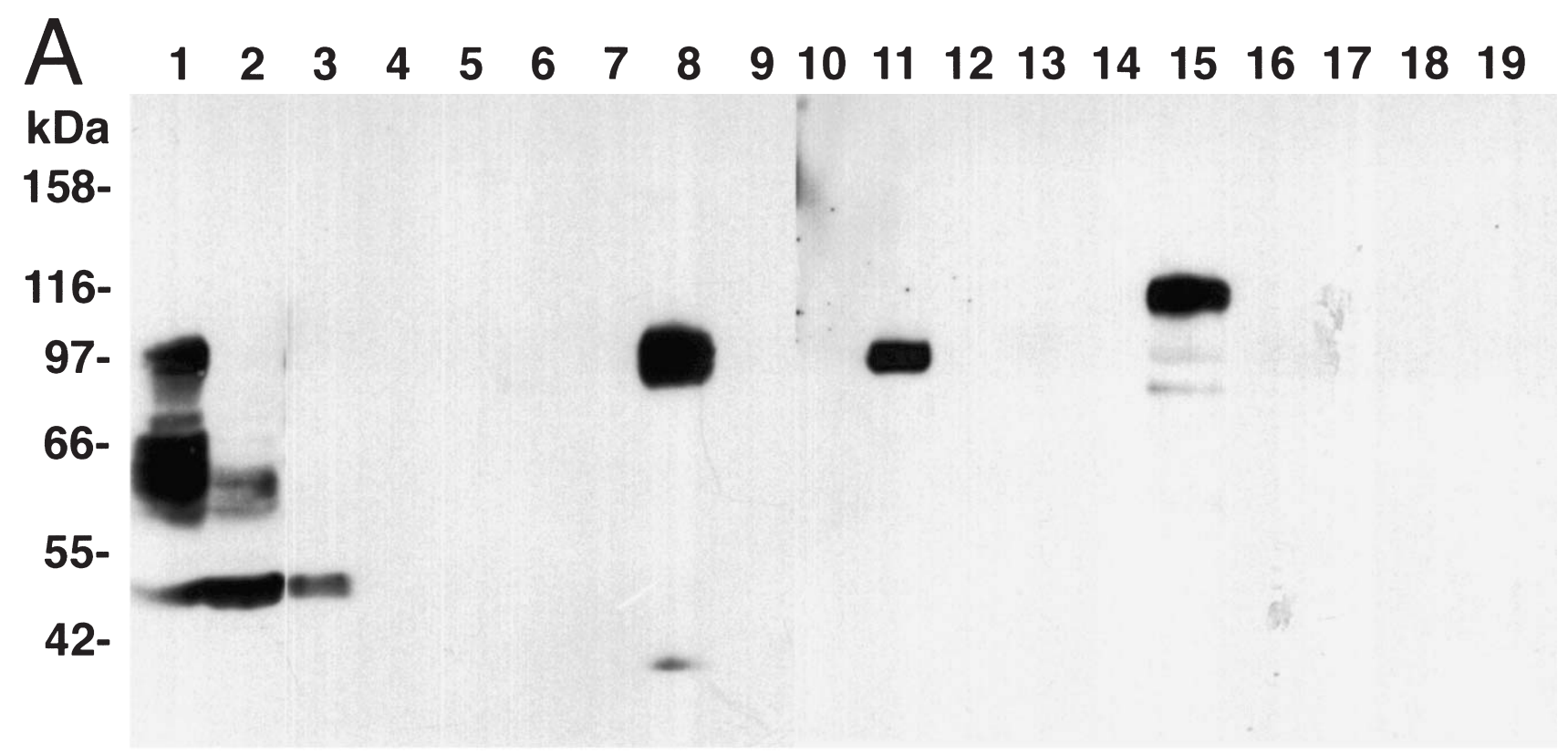

B

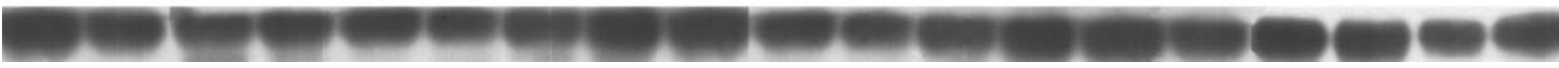

Fig. 1. Western blot analysis showing the expression of $\operatorname{SgIGSF}(\mathbf{A})$ and $\alpha$-tubulin (B) in the cerebrum (lane 1), cerebellum (2), sciatic nerve (3), skeletal muscle (4), heart (5), thymus (6), spleen (7), lung (8), stomach (9), jejunum (10), liver (11), pancreas (12), kidney (13), adrenal gland (14), testis (15), ovary (16), epididymis (17), seminal vesicle (18) and uterus (19). Each lane contains tissue lysate equivalent to $20 \mu \mathrm{g}$ proteins. Molecular weights $(\mathrm{kDa})$ of proteins are indicated. 

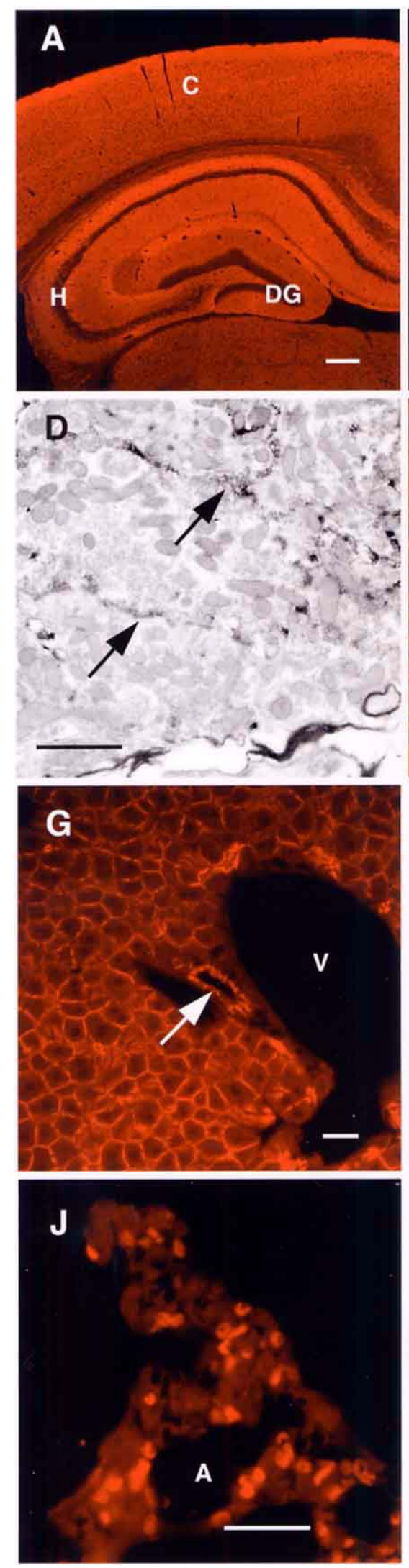
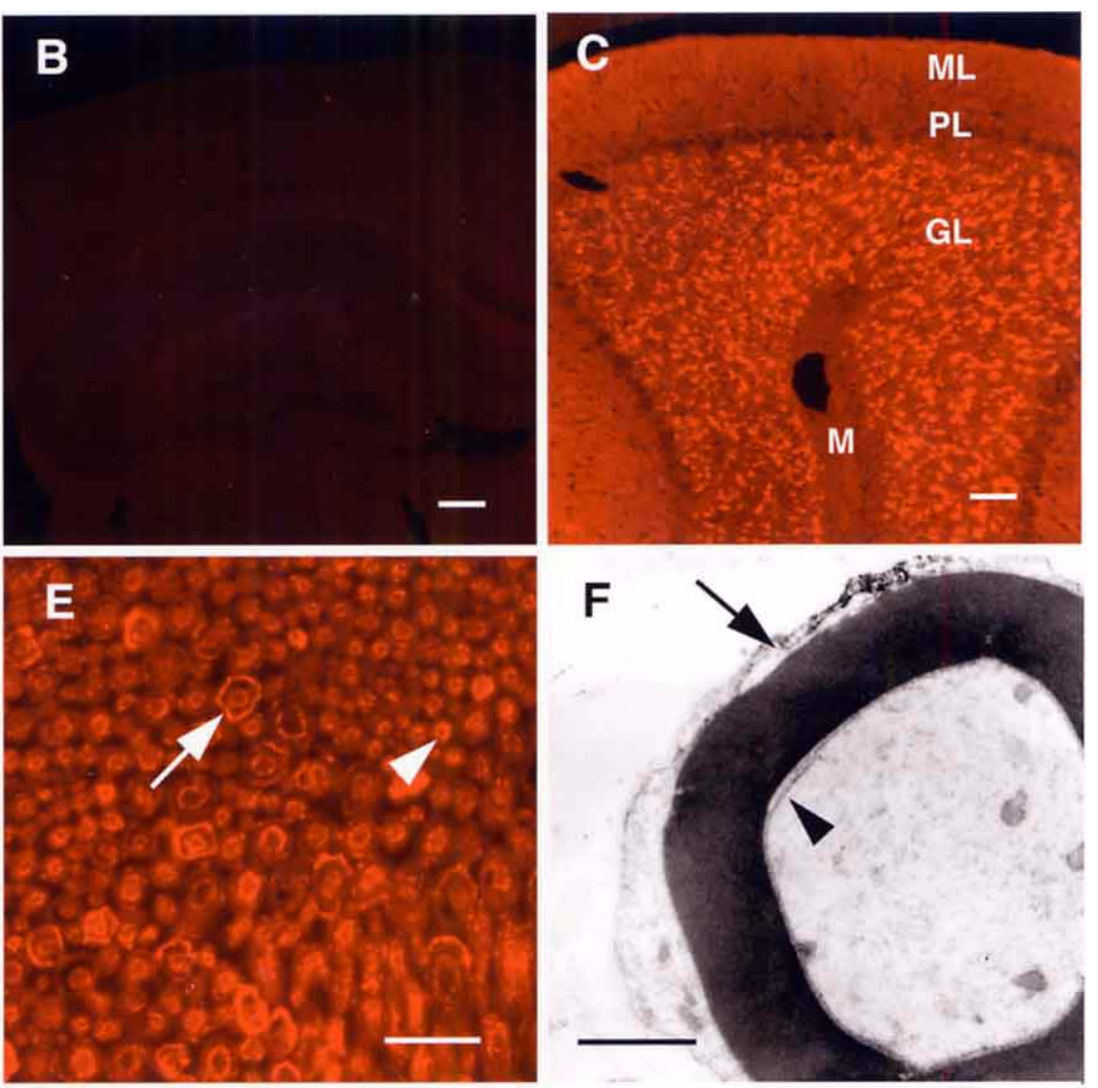

H.

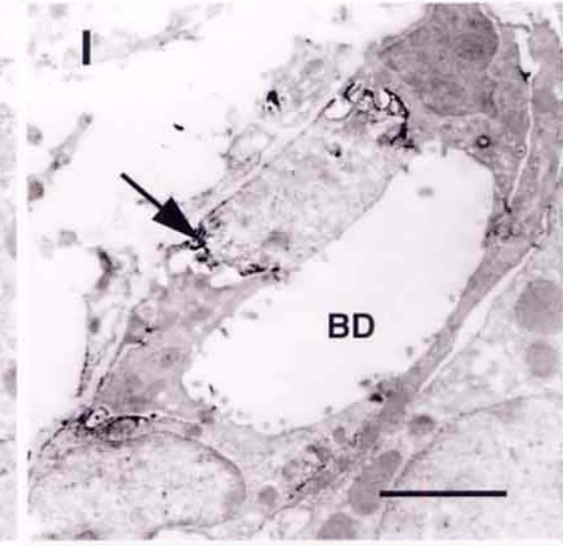

K

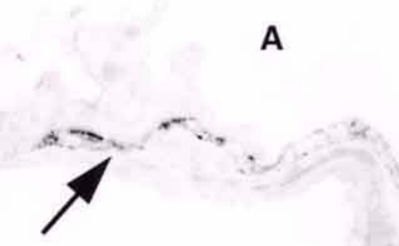

A

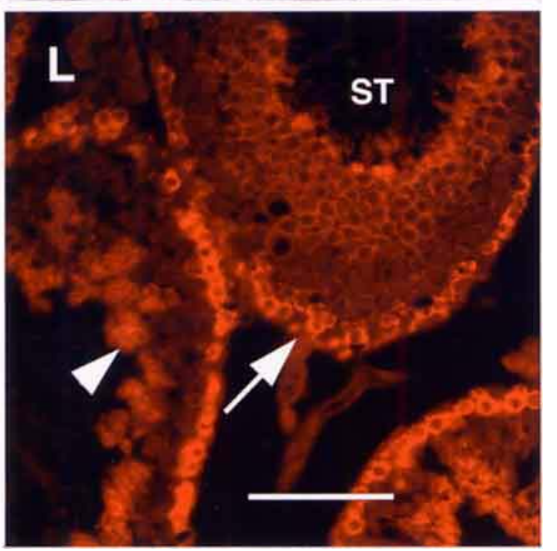

Fig. 2 
membrane portions of apposing hepatocytes and of biliary epithelial cells, whereas the membrane portions facing the microbiliary lumen and Space of Disse in the hepatocytes or the biliary lumen and basal lamina in the biliary epithelial cells were devoid of the immunoreaction (Fig. 2H, I).

In the lung, SgIGSF immunoreactivity was localized to the boundary between the epithelial cells lining the alveoli and bronchioles (Fig. 2J). Electron microscopy also demonstrated the lateral membrane portion, but not the apical or basal membrane portion, of these epithelial cells to be immunoreactive for SgIGSF (Fig. 2K).

In the testis, the spermatogenic cells located in the basal and apical portions of the seminiferous tubules, but not Sertoli cells, were immunoreactive for SgIGSF (Fig. 2L), as reported previously [23].

Similar to the cerebrum, all other organs and tissues were negative for immunostaining using the antibody absorbed with the specific blocking polypeptide as control (data not shown).

\section{Discussion}

In the present study, Western blot analysis has demonstrated the expression of SgIGSF in the liver, lung and nervous system, in addition to the testis that we previously reported [22]. The number of predicted amino acid residues of SgIGSF is 445, which is equivalent to putative molecular weight $52 \mathrm{kDa}$, including $5 \mathrm{kDa}$ for 41 amino acid residues in the signal peptide. However, the apparent molecular weight of SgIGSF on Western blotting in the present study varies from organ to organ with multiple protein bands in the cerebrum, cerebellum and testis. The $45-\mathrm{kDa}$ band shown in all nervous tissues examined may represent the pure polypeptide, which may be the dominant form of SgIGSF in the sciatic nerve. On the other hand, we previously demonstrated in the testis that SgIGSF is a glycoprotein, and that its heterogeneity in size is due to the pattern of glycosylation that shows changes with the postnatal age [23]. As SgIGSF possesses five sites of $\mathrm{N}$-glycosylation on its extracellular domain, different numbers and combinations of the sites may be glycosylated in different cell types. Such patterns of glycosylation of SgIGSF may be associated with different roles of SgIGSF in different organs and tissues.

In the liver and lung, immunohistochemical analysis has demonstrated that SgIGSF is localized to the lateral membrane portions of apposing epithelial cells of the same type, whereas the apical and basal membrane portions of these cells are devoid of SgIGSF. In general, various adhesion molecules, including the members of IGSF, are known to bind to each other (homophilic binding) and/or to other molecules (heterophilic binding) [3]. In vitro study showed that SgIGSF undergoes homophilic binding in a $\mathrm{Ca}^{2+}$ independent manner [13]. Since SgIGSF in the liver and lung is present on the apposing membranes of the same cell types, it is likely to be involved primarily in the homophilic binding, although another possibility is not ruled out that some other adhesion molecule forms the heterophilic binding with SgIGSF between the apposing cell membranes. As there is no indication for ultrastructural localization of SgIGSF in the specific junctional devices such as tight junction, adherent junction and desmosome, it probably functions in the adhesion of epithelial cells on the entire surface of apposing cell membranes. Such a role of SgIGSF as homophilic adhesion molecule is consistent with the recent in vitro studies indicating that SgIGSF causes suppression of the malignancy of lung cancer cell lines [12] and also aggregation of mast cells [11].

In the central nervous system represented by the cerebrum and cerebellum, the observation of intense immunoreactivity on the neuronal membranes at some synaptic sites is consistent with the previous report on the pivotal role of homophilic binding of SgIGSF in the synapse formation by cultured neurons [2]. However, judging from rather diffuse distribution of immunoreactivity all over the gray and white matters of the central nervous system, SgIGSF may also be present in the dendrites and axons remote from the synaptic sites, as well as in the glial cells. Detailed ultrastructural studies on the cellular and subcellular localization of SgIGSF in the central nervous system will be necessary to clarify this issue. In the peripheral nervous system represented by the sciatic nerve, SgIGSF immunoreactivity is present all over the axolemma and the membrane at the outer surface of Schwann cells. Although the inside of the myelin sheath is immunonegative, it is not clear if the inner surface of myelin sheath is immunopositive for SgIGSF. These results raise a possibility that SgIGSF functions as adhesion molecule not only in the neuron-to-neuron but also in the neuron-to-glia and gliato-glia interactions. It is also of interest to determine if the binding of SgIGSF in these interactions, if any, is homophilic or heterophilic in nature.

In the testis, we previously performed a detailed study

Fig. 2. Light and electron microscopic immunohistochemistry for SgIGSF in adult mouse cerebrum (A, B), cerebellum (C, D), sciatic nerve (E, F), liver (G, H, I), lung (J, K) and testis (L). (A) C, cortex; H, hippocampus; DG, dental gyrus. (B) The primary antibody has been absorbed with SgIGSF polypeptide. (C) ML, molecular layer; PL, Purkinje cell layer; GL, granular layer; M, medulla. (D) Arrows, immunoreactivity on the apposing membranes of neurons in the granular layer. (E) Arrow, Schwann cell; arrowhead, axon. (F) Arrow, immunoreactivity on Schwann cell membrane; arrowhead, immunoreactivity on axolemma. (G) V, interlobular vein; arrow, interlobular bile duct. (H) BC, bile canaliculus; SD, Space of Disse; arrow, immunoreactivity on the apposing membrane portions of hepatocytes. (I) BD, bile duct; arrow, immunoreactivity on the apposing membrane portions of bile duct epithelial cells. (J) A, alveolus. (K) A, alveolus; arrow, immunoreactivity on the apposing membrane portions of alveolar epithelial cells. (L) ST, seminiferous tubule; arrow, spermatogonia and spermatocytes; arrowhead, elongating spermatids. Bars $=100 \mu \mathrm{m}(\mathbf{A}, \mathbf{B}, \mathbf{C}, \mathbf{L}) ; 25 \mu \mathrm{m}(\mathbf{E}, \mathbf{G}, \mathbf{J}) ; 1 \mu \mathrm{m}(\mathbf{D}, \mathbf{F}, \mathbf{H}, \mathbf{I})$. 
and revealed that SgIGSF is localized to the membranes of intermediate spermatogonia through early pachytene spermatocytes and also of the elongating spermatids at step 7 and later, whereas the membrane of Sertoli cells is devoid of SgIGSF [23]. Furthermore, SgIGSF was shown to bind to the surface of Sertoli cells, suggesting that SgIGSF on the spermatogenic cells primarily undergoes the heterophilic binding with some other molecule on Sertoli cells and thereby plays a significant role in spermatogenesis. However, the homophilic binding between the spermatogenic cells through SgIGSF may also exist.

In the present study, it is notable that the lung and liver, wherein SgIGSF seems to function primarily in the homophilic binding, produce SgIGSF with single molecular weight, whereas the nervous system and testis, wherein SgIGSF may function in both the homophilic and heterophilic bindings, produce SgIGSF with multiple molecular weights. In this connection, a recent study has indicated that SgIGSF produced by mast cells in the connective tissue is responsible for both the homophilic binding in the interaction among mast cells and the heterophilic binding in the interaction between mast cells and fibroblasts, and that it also has multiple molecular weights [11].

It is reported that some IGSF molecules play a role in signal transduction in addition to one in cellular binding. SgIGSF has a short cytoplasmic domain of 46 amino acid residues, which displays high similarity with human glycophorin $\mathrm{C}$, human contactin-associated protein 2 (Caspr2), human TSLC1-like gene 1 (TSLL1), TSLL2, human syndecan-2, rat paranodin and Drosophila Neurexin IV [7]. The carboxyl terminus of these IGSF proteins possesses the consensus sequence of type II PDZ binding motif (Tyr/PhePhe-Ile). In the Nectin family, this PDZ binding motif in the cytoplasmic domain interacts with actin filaments via afadin, a nectin- and actin-binding protein [18], and may be involved in signal transduction. As SgIGSF also has PDZ binding motif, Tyr-Phe-Ile, in the carboxyl terminus [21], it will be of primary interest to explore the possible role of SgIGSF in signal transduction.

The present study has suggested that SgIGSF plays multiple biological roles as an adhesion molecule in a variety of adult mouse organs and tissues.

\section{Acknowledgments}

We thank Shuichi Yamazaki for his histological and photographic works. This work was supported by Grant-inAid for Scientific Research from the Ministry of Education, Science and Culture of Japan to TW.

\section{References}

1. Balda, M. S. and Matter, K. (2003) Epithelial cell adhesion and the regulation of gene expression. Trends Cell Biol. 13; 310-318.

2. Biederer, T., Sara, Y., Mozhayeva, M., Atasoy, D., Liu, X., Kavalali, E. T. and Sudhof, T. C. (2002) SynCAM, a synaptic adhesion molecule that drives synapse assembly. Science 297;
$1525-1531$.

3. Brummendorf, T. and Rathjen, F. G. (1995) Cell adhesion molecule 1: immunoglobulin superfamily. Protein Profile 2; 963-1108.

4. Crossin, K. L. and Krushel, L. A. (2000) Cellular signaling by neural cell adhesion molecules of the immunoglobulin superfamily. Dev. Dyn. 218; 260-279.

5. de Bono, B. and Chothia, C. (2003) Exegesis: a procedure to improve gene predictions and its use to find immunoglobulin superfamily proteins in the human and mouse genomes. Nucleic Acids Res. 31; 6096-6103.

6. Edelman, G. M. (1986) Cell adhesion molecules in the regulation of animal form and tissue pattern. Annu. Rev. Cell Biol. 2; 81116.

7. Fukuhara, H., Kuramochi, M., Nobukuni, T., Fukami, T., Saino, M., Maruyama, T., Nomura, S., Sekiya, T. and Murakami, Y. (2001) Isolation of the TSLL1 and TSLL2 genes, members of the tumor suppressor TSLC1 gene family encoding transmembrane proteins. Oncogene 20; 5401-5407.

8. Gumbiner, B. M. (1996) Cell adhesion: the molecular basis of tissue architecture and morphogenesis. Cell 84; 345-357.

9. Hortsch, M. (2000) Structural and functional evolution of the L1 family: are four adhesion molecules better than one? Mol. Cell. Neurosci. 15; 1-10.

10. Hynes, R. O. (1996) Targeted mutations in cell adhesion genes: what have we learned from them? Dev. Biol. $180 ; 402-412$.

11. Ito, A., Jippo, T., Wakayama, T., Morii, E., Koma, Y., Onda, H., Nojima, H., Iseki, S. and Kitamura, Y. (2003) SgIGSF: a new mast-cell adhesion molecule used for attachment to fibroblasts and transcriptionally regulated by MITF. Blood 101; 2601-2608.

12. Kuramochi, M., Fukuhara, H., Nobukuni, T., Kanbe, T., Maruyama, T., Ghosh, H. P., Pletcher, M., Isomura, M., Onizuka, M., Kitamura, T., Sekiya, T., Reeves, R. H. and Murakami, Y. (2001) TSLC1 is a tumor-suppressor gene in human non-smallcell lung cancer. Nat. Genet. 27; 427-430.

13. Masuda, M., Yageta, M., Fukuhara, H., Kuramochi, M., Maruyama, T., Nomoto, A. and Murakami, Y. (2002) The tumor suppressor protein TSLC1 is involved in cell-cell adhesion. J. Biol. Chem. 277; 31014-31019.

14. Nelson, W. J. and Nusse, R. (2004) Convergence of Wnt, betacatenin, and cadherin pathways. Science $303 ; 1483-1487$.

15. Rutishauser, U. (2000) Defining a role and mechanism for IgCAM function in vertebrate axon guidance. J. Cell Biol. 149; 757-760.

16. Schuch, U., Lohse, M. J. and Schachner, M. (1989) Neural cell adhesion molecules influence second messenger systems. Neuron $3 ; 13-20$.

17. Sutherland, J. D. and Witke, W. (1999) Molecular genetic approaches to understanding the actin cytoskeleton. Curr. Opin. Cell Biol. 11; 142-151.

18. Takai, Y. and Nakanishi, H. (2003) Nectin and afadin: novel organizers of intercellular junctions. J. Cell Sci. 116; 17-27.

19. Takeichi, M. (1995) Morphogenetic roles of classic cadherins. Curr. Opin. Cell Biol. 7; 619-627.

20. Vestweber, D. and Blanks, J. E. (1999) Mechanisms that regulate the function of the selectins and their ligands. Physiol. Rev. 79; $181-213$

21. Wakayama, T., Ohashi, K., Mizuno, K. and Iseki, S. (2001) Cloning and characterization of a novel mouse immunoglobulin superfamily gene expressed in early spermatogenic cells. Mol. Reprod. Dev. 60; 158-164.

22. Wakayama, T., Hamada, K., Yamamoto, M., Suda, T. and Iseki, S. (2003) The expression of platelet endothelial cell adhesion molecule-1 in mouse primordial germ cells during their migration and early gonadal formation. Histochem. Cell Biol. 119; 355362.

23. Wakayama, T., Koami, H., Ariga, H., Kobayashi, D., Sai, Y., 
Tsuji, A., Yamamoto, M. and Iseki, S. (2003) Expression and functional characterization of the adhesion molecule spermatogenic immunoglobulin superfamily in the mouse testis. Biol. Reprod. 68; 1755-1763.

24. Williams, A. F. and Barclay, A. N. (1988) The immunoglobulin superfamily-domains for cell surface recognition. Annu. Rev. Immunol. 6; 381-405.
25. Yagi, T. and Takeichi, M. (2000) Cadherin superfamily genes: functions, genomic organization, and neurologic diversity. Genes Dev. 14; 1169-1180.

26. Yamamoto, M. and Iseki, S. (2003) Co-expression of NGF and its high-affinity receptor TrkA in the rat carotid body chief cells. Acta Histochem. Cytochem. 36; 377-383. 